\title{
Fatal infections in older patients with inflammatory bowel disease on anti-tumor necrosis factor therapy
}

\author{
Way-Seah Lee ${ }^{1,2}$, Najib Azmi $^{3,4}$, Ruey-Terng Ng${ }^{1}$, Sik-Yong Ong ${ }^{1}$, Sasheela Sri La Ponnampalavanar ${ }^{3}$, \\ Sanjiv Mahadeva ${ }^{3}$, Ida Hilmi ${ }^{3}$ \\ ${ }^{I}$ Department of Paediatrics, ${ }^{2}$ University Malaya Paediatrics and Child Health Research Group, and ${ }^{3}$ Department of Medicine, University of \\ Malaya Faculty of Medicine, Kuala Lumpur, ${ }^{4}$ Department of Medicine, University Sains Islam Malaysia Faculty of Medicine, Kuala Lumpur, \\ Malaysia
}

Anti-tumor necrosis factor (anti-TNF) is highly effective in inflammatory bowel disease (IBD); however, it is associated with an increased risk of infections, particularly in older adults. We reviewed 349 patients with IBD, who were observed over a 12-month period, 74 of whom had received anti-TNF therapy ( 71 patients were aged $<60$ years and 3 were aged $\geq 60$ years). All the 3 older patients developed serious infectious complications after receiving anti-TNFs, although all of them were also on concomitant immunosuppressive therapy. One patient developed disseminated tuberculosis, another patient developed cholera diarrhea followed by nosocomial pneumonia, while the third patient developed multiple opportunistic infections (Pneumocystis pneumonia, cryptococcal septicemia and meningitis, Klebsiella septicemia). All 3 patients died within 1 year from the onset of the infection(s). We recommend that anti-TNF, especially when combined with other immunosuppressive therapy, should be used with extreme caution in older adult patients with IBD. (Intest Res 2017;15:524-528)

Key Words: Elderly patients; Anti-tumor necrosis factor; Serious infections

\section{INTRODUCTION}

Since their first introduction in 1998, tumor necrosis factor inhibitor (anti-TNF) therapies have revolutionized the medical treatment of IBD. ${ }^{1-5}$ Many current guidelines recommend the use of anti-TNF in adult patients with moderate-to-severe CD and UC refractory to corticosteroid (CS), ${ }^{6-10}$ as well as in pediatric patients with moderate-to-severe $\mathrm{CD}$ despite optimum immunomodulator therapy. ${ }^{11}$ However, concerns about the safety of anti-TNF, particularly the risk of infection and malignancy, persist. ${ }^{12}$ Several studies have shown that

Received June 5, 2017. Revised August 16, 2017.

Accepted August 18, 2017.

Correspondence to Way-Seah Lee, Department of Paediatrics, University Malaya Medical Center, University of Malaya Faculty of Medicine, Kuala

Lumpur 59100, Malaysia. Tel: +603-7949-2065, Fax: +603-7949-4704,

E-mail: leews@ummc.edu.my

Financial support: The present research was funded by a research grant from the Ministry of Higher Education, Malaysia (UM.C/625/HIR/MOHE/ CHAN/13/1). Conflict of interest: None. age is an important risk factor for infection in patients on anti-TNF therapy, ${ }^{13,14}$ although the magnitude of this risk remains difficult to quantify.

To assess this risk, we retrospectively reviewed the medical records of 349 patients with IBD (276 adults [215 patients aged $<60$ years, 61 patients aged $\geq 60$ years] and 73 children) who were followed up at University Malaya Medical Center, Malaysia in the last 10 years. Among the patients, 186 (53\%) were male and 163 (47\%) were female. Approximately half of the patients had UC (n=187, 54\%), 156 (45\%) had CD, and 6 (2\%) had IBD-unspecified. Seventy-four patients (21\%) received anti-TNF therapy.

In patients who were not exposed to anti-TNFs, the risk of non-IBD-related significant infectious complications was surprisingly very low, where 2 patients $(0.7 \%)$ under the age of 60 years ( 1 adult and 1 child) developed pulmonary tuberculosis (TB). No patient in the older adult age group developed any significant infectious complications.

In the group of patients who received anti-TNF, however,

\footnotetext{
๑ Copyright 2017. Korean Association for the Study of Intestinal Diseases. All rights reserved.

This is an Open Access article distributed under the terms of the Creative Commons Attribution Non-Commercial License (http://creativecommons.org/licenses/by-nc/4.0)

which permits unrestricted non-commercial use, distribution, and reproduction in any medium, provided the original work is properly cited.
} 
serious non-IBD-related infectious complications were observed in $6(8.5 \%)$ of the 71 patients in the younger group and all 3 patients (100\%) in the elderly group. In the younger age group, 2 patients developed pulmonary TB, 2 patients developed pneumonia, and 1 patient had Escherichia coli septicemia. Six other patients $(8.5 \%)$ in the younger age group developed mild shingles. One patient died of nosocomial pneumonia; however, this patient had a background of refractory small-bowel CD with short bowel syndrome, complicated by an iatrogenic pneumothorax following a Hickman line insertion. All the other patients recovered well after treatment.

In contrast, all 3 patients ( $100 \%$ ) aged $\geq 60$ years developed serious infections: 1 patient developed disseminated TB, another patient succumbed following nosocomial pneumonia and Vibrio cholerae infection, and the third patient developed multiple opportunistic infections (Pneumocystis pneumonia and cryptococcal meningitis). All 3 patients in the older age group died within 1 year from the onset of infection(s). We describe these cases in detail. The present study was approved by the Institutional Ethical Committee of UMMC (MECID: 20157-1485).

\section{CASE REPORTS}

\section{Case 1 (Multiple Opportunistic Infections)}

A 70-year-old woman with a background of CD of the terminal ileum for 21 years. She had undergone limited right hemicolectomy, 20 years prior to presentation; however, she experienced postoperative recurrence of the condition, for which she was on azathioprine $50 \mathrm{mg}$ and prednisolone 5 $\mathrm{mg}$ for 10 years. Despite this, she presented with a history of worsening abdominal pain and diarrhea. After undergoing the standard screening test for TB (chest X-ray [CXR] and tuberculin skin test), she was commenced on 3 doses of infliximab (IFX). However, 4 weeks following the third infusion, she presented with persistent pyrexia of unknown origin. She was initially treated with intravenous cefuroxime but continued to be pyrexial. Blood cultures grew Cryptococcal neoformans as well as Klebsiella pneumoniae. Cerebrospinal fluid examination also confirmed the presence of cryptococcal antigen. The patient was initially treated with amphotericin $\mathrm{B}$, which was then switched to voriconazole. She was also commenced on intravenous cefuroxime and metronidazole. After 2 weeks, however, she presented with increasing shortness of breath and was found to be hypoxic. A repeat CXR revealed mild interstitial shadowing. CT image of the chest revealed pneumonia of the right lung and multiple shadows (Fig. 1). The patient was diagnosed by infectious disease specialists with possible Pneumocystis jiroveci pneumonia, for which she was commenced on co-trimoxazole. She had a complicated course, but she was finally discharged after 4 weeks on prednisolone $10 \mathrm{mg}$ and co-trimoxazole. She appeared to be stable 1 month after discharge. However, she was readmitted 3 months later owing to relapse of her diarrhea. Blood culture during this admission showed no organisms; moreover, her stool was negative, even for Clostridium difficile. She was treated with intravenous hydrocortisone. However, she developed sudden cardiorespiratory arrest and died. A post-mortem was not carried out.

\section{Case 2 (Disseminated Tuberculosis)}

A 68-year-old man with a history of $\mathrm{CD}$ of the terminal ileum for 21 years. He had undergone 4 previous surgeries for CD of the terminal ileum. His condition recurred after the operation (abdominal pain, diarrhea, and weight loss), which was confirmed endoscopically (Fig. 2). He was initially commenced on azathioprine $50 \mathrm{mg}$ a day and prednisolone $40 \mathrm{mg}$ a day; however, he failed to achieve clinical remission after 4 weeks of therapy. IFX was added owing to a suboptimal response and the history of multiple surgeries. Pre-therapy screening for TB (CXR and T-SPOT. TB test) was negative, and the patient received 3 infusions of IFX. His CS was tapered after the first infusion of IFX by $5 \mathrm{mg} / \mathrm{wk}$, as per our general protocol. He responded very well to the treatment and achieved clinical remission. However, 3 days after his third infusion, he presented with pyrexia of unknown ori-

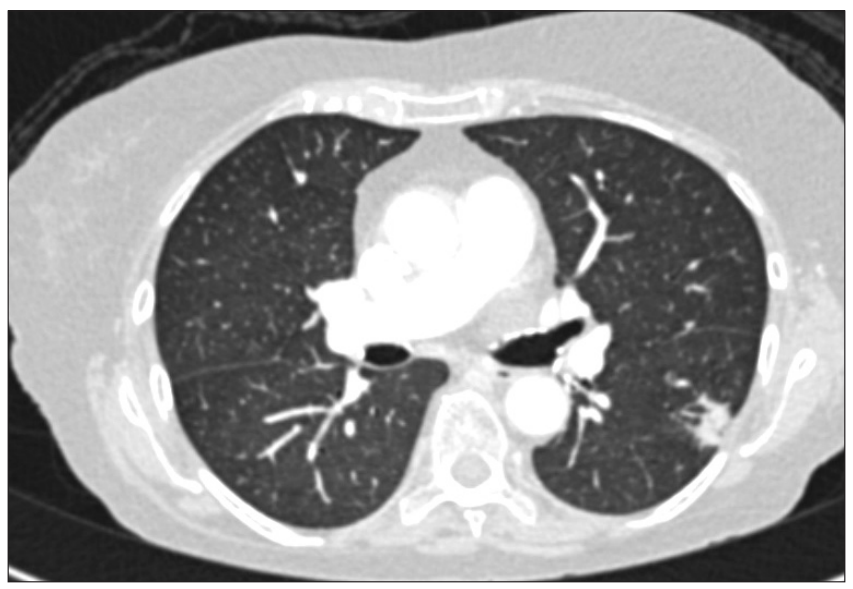

Fig. 1. Cross-sectional view of the CT image showing pneumonia of the right lung and multiple alveolar consolidations, consistent with the diagnosis of Pneumocystis jiroveci pneumonia (case 1). 


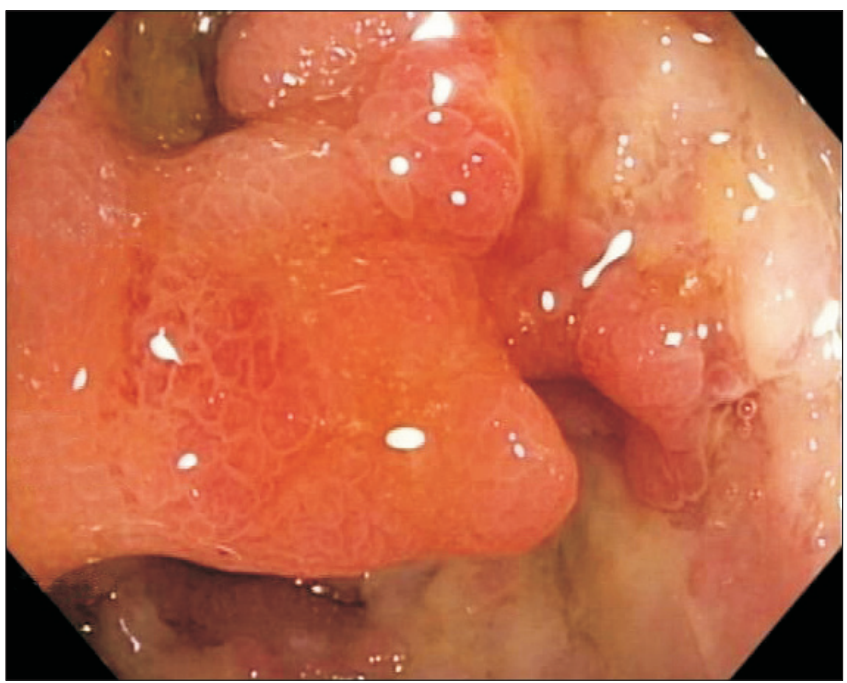

Fig. 2. Endoscopic image showing recurrence of CD (case 2).

gin. He was initially treated with intravenous ceftriaxone but failed to respond and was transferred to the intensive care unit (ICU) with septicemic shock and multiorgan failure. CT imaging of the abdomen revealed disseminated microabscesses of the liver and spleen (Fig. 3). Biopsy of the liver abscess showed the presence of AFB, confirming the diagnosis of TB and active CD. Unfortunately, this patient also experienced bleeding after splenic biopsy and underwent a laparotomy to secure hemostasis. He had a prolonged stay in the ICU (4 weeks), but was eventually discharged. However, he remained unwell after discharge, with persistent diarrhea from active $\mathrm{CD}$ and progressive weight loss from ongoing active TB. He was on a small maintenance dose of prednisolone (10 mg) and anti-TB treatment, but azathioprine was discontinued owing to neutropenia. Although he continued to have symptoms of $\mathrm{CD}$, he was deemed unfit for surgery. The patient was admitted 8 months later with worsening symptoms (diarrhea, abdominal pain, and weight loss), and stool culture during this admission grew nontyphoidal Salmonella. He was provided nutritional support and antibiotic therapy. However, he developed nosocomial pneumonia, and despite treatment with intravenous piperacillin/tazobactam, he died 2 weeks later.

\section{Case 3 (Nosocomial Pneumonia and V. cholerae Enteritis)}

A 61-year-old woman with Crohn's colitis for 6 years. She had chronic active disease despite being on maintenance therapy with azathioprine and sulfasalazine, requiring multiple courses of prednisolone. She subsequently developed a perianal fistula as well as a colovesical fistula, with recur-

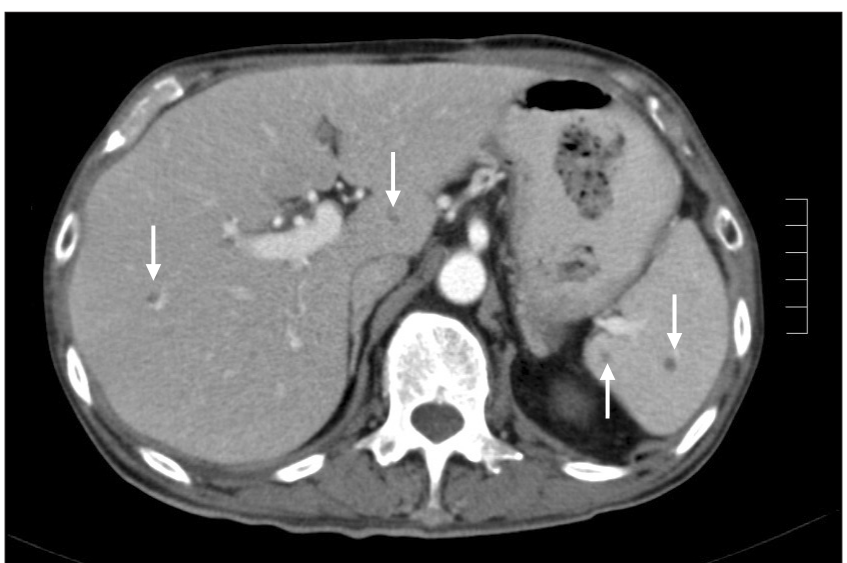

Fig. 3. CT image of the abdomen showing disseminated micro-abscesses of the liver and spleen (arrows) (case 2).

rent urinary tract infections. Subsequently, it was decided to start her on IFX. She initially showed remarkable improvement; however, 5 weeks after her fourth dose, she developed bloody diarrhea and discharge from the perianal fistula. A colonoscopy was not carried out to rule out cytomegalovirus (CMV) colitis. Owing to the flare of her CD, the patient was started on intravenous hydrocortisone while awaiting stool cultures. However, empirical antibiotics were not commenced as she had no fever and the clinical presentation was similar to that during her previous flares. However, her stool culture subsequently isolated $V$. cholera and she was also commenced on tetracycline. She improved clinically and was discharged after 5 days. However, she was re-admitted 3 days later owing to shortness of breath. On this admission, she was in septicemic shock and respiratory distress, requiring intubation. Chest radiograph showed widespread lung consolidation suggestive of atypical pneumonia. The patient subsequently died as a result of severe bronchopneumonia despite aggressive treatment with intravenous piperacillin/tazobactam.

\section{DISCUSSION}

Biologics, including anti-TNF, has become the standard of care in the management of patients with IBD. ${ }^{2,3}$ Infections and malignancy are well recognized serious complications associated with this therapy. However, most long-term registries studying the safety of anti-TNF, such as the TREAT registry, found a low overall risk of $1.7 \%{ }^{15}$ However, this finding does not apply to older patients. An Italian study involving 95 patients with IBD found that among patients older than 65 years who were treated with TNF inhibitors, $11 \%$ developed 
severe infections, $3 \%$ developed neoplasms, and $10 \%$ died. $^{14}$ Similarly, another study also found that the risk for any severe adverse events was significantly higher in patients aged $\geq 65$ years compared to the risk in patients aged $<65$ years who receive anti-TNF (risk ratio, $4.7 ; P<0.001$ ).${ }^{16}$ In a study evaluating the risk factors for infection in patients with IBD, it was found that the RR of opportunistic infections was the highest in patients with IBD who were aged more than 50 years (OR, 3.0; 95\% CI, 1.2-7.2; relative to those aged 24 years or younger) ${ }^{16}$

Our report further adds to the growing evidence that there is significant risk of life-threatening infections in patients the older age group who receive anti-TNF therapy, even in the absence of co-morbidities. In our study, all 3 patients in the older age group developed life-threatening infections after receiving anti-TNF, and died within a year of the development of these infections. Similarly, our study also confirms previous findings that the use of anti-TNF is relatively safe in the younger age group, with a low risk of serious complications.

There were several learning points to highlight from the study. Firstly, although all 3 of the older adult patients were screened for latent $\mathrm{TB}$, they did not receive the recommended vaccines prior to commencing therapy. Although there was no evidence that they developed any of the vaccine-preventable diseases, there may have been a possible protective effect if the patients had received at least the influenza and pneumococcal vaccines prior to anti-TNF therapy. Unfortunately, routine vaccination prior to anti-TNF therapy was only regularly implemented in our setting in the last 5 years. None of the patients who have had previous exposure to varicella zoster received the shingles vaccine, as it is expensive and not readily available in Malaysia. This is worth noting, as there were 6 cases of shingles in the younger age group.

Second, all 3 of the patients in the older age group had received triple immunosuppressive therapy. All patients were on thiopurines, which is the standard maintenance therapy in CD; however, they were also exposed to CS during the development of the infections. Case 1 patient was kept on a small dose of prednisolone as she was steroid dependent, Case 2 patient was on a tapering dose of prednisolone, and Case 3 patient had been started on intravenous hydrocortisone as "rescue" therapy when she was admitted for exacerbation of CD. The study of Toruner et al. ${ }^{13}$ has clearly shown that the risk of infectious complications increases exponentially with an increasing number of immunosuppressive medications, and it is likely that this was a major contributing factor to the adverse outcomes. ${ }^{16}$ In addition, the patients were likely to be severely malnourished owing to their active CD.

In conclusion, the benefits of starting anti-TNF therapy in older adult patients must strongly be weighed against the very significant risks highlighted in the study. However, if anti-TNF therapy is to be commenced in these patients, it is imperative that the recommended vaccines are administered, particularly influenza, zoster, and pneumococcal. The patients should also receive aggressive concomitant nutritional support; thiopurines and CS should be discontinued, with rapid tapering of CS, if necessary. Ideally, gut-specific biologic therapies such as vedolizumab, which has a more favorable side-effect profile in terms of systemic complications, might be a better choice in older adult patients.

In summary, our study shows that there is a significant risk of life-threatening infections among older adult patients with IBD who receive anti-TNF therapy, especially when combined with other immunosuppressive therapy. Anti-TNF has to be used with extreme caution in older patients with IBD.

\section{AUTHOR CONTRIBUTION}

W.S.L. is the guarantor of the manuscript.

\section{REFERENCES}

1. Dignass A, Van Assche G, Lindsay JO, et al. The second European evidence-based consensus on the diagnosis and management of Crohn's disease: current management. J Crohns Colitis 2010;4:28-62.

2. Dignass A, Lindsay JO, Sturm A, et al. Second European evidence-based consensus on the diagnosis and management of ulcerative colitis part 2: current management. J Crohns Colitis 2012;6:991-1030.

3. Hanauer SB, Feagan BG, Lichtenstein GR, et al. Maintenance infliximab for Crohn's disease: the ACCENT I randomised trial. Lancet 2002;359:1541-1549.

4. Sandborn WJ, Hanauer SB, Rutgeerts P, et al. Adalimumab for maintenance treatment of Crohn's disease: results of the CLASSIC II trial. Gut 2007;56:1232-1239.

5. Schreiber S, Khaliq-Kareemi M, Lawrance IC, et al. Maintenance therapy with certolizumab pegol for Crohn's disease. N Engl J Med 2007;357:239-250. 
6. D'Haens GR, Panaccione R, Higgins PD, et al. The London Position Statement of the World Congress of Gastroenterology on Biological Therapy for IBD with the European Crohn's and Colitis Organization: when to start, when to stop, which drug to choose, and how to predict response? Am J Gastroenterol 2011;106:199-212.

7. Mowat C, Cole A, Windsor A, et al. Guidelines for the management of inflammatory bowel disease in adults. Gut 2011;60:571607.

8. Ueno F, Matsui T, Matsumoto T, et al. Evidence-based clinical practice guidelines for Crohn's disease, integrated with formal consensus of experts in Japan. J Gastroenterol 2013;48:31-72.

9. Park JJ, Yang SK, Ye BD, et al. Second Korean guidelines for the management of Crohn's disease. Intest Res 2017;15:38-67.

10. Choi CH, Moon W, Kim YS, et al. Second Korean guidelines for the management of ulcerative colitis. Intest Res 2017;15:7-37.

11. Ruemmele FM, Veres G, Kolho KL, et al. Consensus guidelines of ECCO/ESPGHAN on the medical management of pediatric Crohn's disease. J Crohns Colitis 2014;8:1179-1207.
12. Targownik LE, Bernstein CN. Infectious and malignant complications of TNF inhibitor therapy in IBD. Am J Gastroenterol 2013;108:1835-1842.

13. Toruner M, Loftus EV Jr, Harmsen WS, et al. Risk factors for opportunistic infections in patients with inflammatory bowel disease. Gastroenterology 2008;134:929-936.

14. Cottone M, Kohn A, Daperno M, et al. Advanced age is an independent risk factor for severe infections and mortality in patients given anti-tumor necrosis factor therapy for inflammatory bowel disease. Clin Gastroenterol Hepatol 2011;9:30-35.

15. Lichtenstein GR, Feagan BG, Cohen RD, et al. Serious infections and mortality in association with therapies for Crohn's disease: TREAT registry. Clin Gastroenterol Hepatol 2006;4:621-630.

16. Lobatón T, Ferrante M, Rutgeerts P, Ballet V, Van Assche G, Vermeire S. Efficacy and safety of anti-TNF therapy in elderly patients with inflammatory bowel disease. Aliment Pharmacol Ther 2015;42:441-451. 